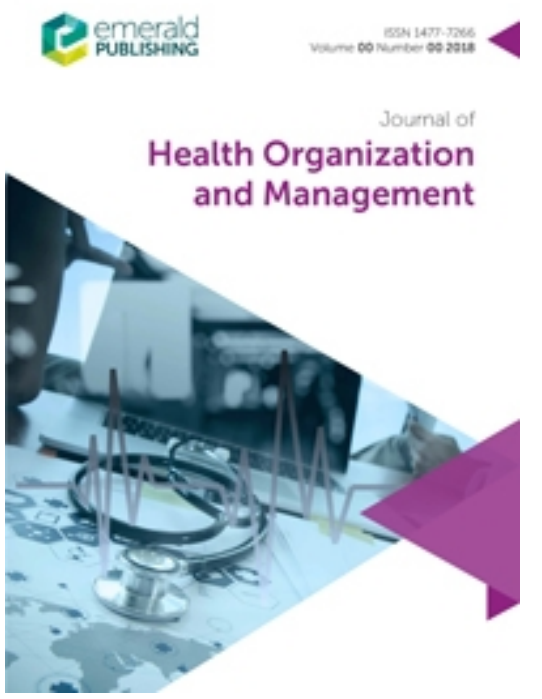

Merger and post-merger integration at Oxford University Hospitals: mixed-methods evaluation and lessons learned

\begin{tabular}{|r|l|}
\hline Journal: & Journal of Health Organization and Management \\
\hline Manuscript ID & JHOM-01-2021-0024.R2 \\
\hline Manuscript Type: & Original Article \\
\hline Keywords: & Mergers, Hospital management, Organizational Culture \\
\hline \multicolumn{2}{l}{} \\
\end{tabular}

SCHOLARONE $^{\text {TM }}$

Manuscripts 
Emerald Group Publishing Limited

Howard House

Wagon Lane

Bingley

West Yorkshire

BD16 1WA

\section{Consent to publish - Release form}

\begin{tabular}{||ll||}
\hline \hline Title of Case & MERGER AND POST-MERGER INTEGRATION AT OXFORD \\
study: & UNIVERSITY HOSPITALS: MIXED-METHODS EVALUATION AND TEN \\
& LESSONS LEARNED \\
\hline \hline & EDWARD J MAILE, DEPARTMENT OF PRIMARY CARE AND PUBLIC \\
& HEALTH, IMPERIAL COLLEGE LONDON, LONDON, UNITED KINGDOM \\
Author: & PAVEL V OVSEIKO, RADCLIFFE DEPARTMENT OF MEDICINE, \\
& UNIVERSITY OF OXFORD, OXFORD, UNITED KINGDOM \\
& SUE DOPSON, SAID BUSINESS SCHOOL, UNIVERSITY OF OXFORD, \\
& OXFORD, UNITED KINGDOM \\
\hline
\end{tabular}

I hereby confirm that I have reviewed the above-entitled case study in full and on behalf of the organisation in question, I provide my full permission for the case study to be published in its entirety for the life of the work in all languages and all formats by Emerald Group Publishing for commercial distribution.

By signing this form, I warrant that I am authorised to grant full permission.

\begin{tabular}{|ll||}
\hline Name: & CHRISTOPHER GOARD \\
\hline \hline Position: & NON-EXECUTIVE DIRECTOR \\
\hline \hline Address: & HEADLEY WAY OXFORD \\
\hline \hline Organisation: & OXFORD UNIVERSITY HOSPITALS NHS FT \\
\hline \hline E-mail: & chris@goard.demon.co.uk \\
\hline
\end{tabular}

Signed:

(either handwritten or insert a scanned image of your signature)

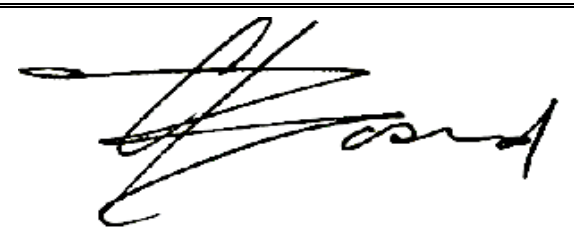

Date reviewed

\& signed: 


\title{
Merger and post-merger integration at Oxford University Hospitals: mixed- methods evaluation and lessons learned
}

\begin{abstract}
Purpose

Hospital mergers are common in the United Kingdom and internationally. However, mergers rarely achieve their intended benefits and are often damaging. This study builds on existing literature by presenting a case study evaluating a merger of two hospitals in Oxford, United Kingdom with three distinct characteristics: merger between two university hospitals; merger between a generalist and specialist hospital; and merger between two hospitals of differing size. In doing so, the study draws practical lessons for other healthcare organisations.
\end{abstract}

\section{Methodology}

Mixed-methods single-case evaluation. Qualitative data from 19 individual interviews and three focus groups were analysed thematically, using constant comparison to synthesise and interpret findings. Qualitative data were triangulated with quantitative clinical and financial data. To maximise research value, the study was co-created with practitioners.

\section{Findings}

The merger was a relative success with mixed improvement in clinical performance and strong improvement in financial and organisational performance. The merged organisation received an improved inspection rating, became debt-free, and achieved Foundation Trust status. The study draws six lessons relating to the contingencies that can make mergers a success: (1) Develop a strong clinical rationale; (2) Communicate the change strategy widely and early; (3) Increase engagement and collaboration at all levels; (4) Be transparent and realistic about the costs and benefits; (5) Be sensitive to the feelings of the other organisation; and (6) Integrate different organizational cultures effectively.

\section{Originality}

This case study provides empirical evidence on the outcome of merger in a university hospital setting. Despite the relatively positive outcome, there is no strong evidence that the benefits could not have been achieved without merger. Given that mergers remain prevalent worldwide, the practical lessons might be useful for other healthcare organisations considering merger. 


\section{Introduction}

Mergers occur when two organisations combine to form a single entity. Usually, motivations include operating synergies, financial synergies and managerial synergies (Petit and Ferris, 2013). Given that mergers in the private sector frequently fail to improve performance (KPMG, 1999; Agrawal and Jaffe, 2001; King et al., 2004; Cartwright and Schoenberg, 2006), alternative motivations include diversification to hedge against declining core business, over-confidence about the benefits, and an individual's desire to gain power or increased remuneration by controlling a larger organisation (Giddy, 2009).

In the United Kingdom (UK), mergers have been a frequent phenomenon in its public health sector for more than twenty years, with 112 of 223 acute National Health Service (NHS) hospital trusts merging between 1997 and 2006 (Gaynor et al., 2012). The creation of Foundation Trust (FT) status in 2002 (Department of Health, 2002), with the desire that all trusts achieved it, gave this trend further impetus (Collins, 2015). FT status confers greater autonomy from government, and if a trust was unlikely to achieve it alone, merger with an existing FT or another trust where the new organisation has a high likelihood of becoming an FT was strongly encouraged (Collins, 2015). There were fifty further NHS mergers between 2010 and 2015 (NHS Improvement, 2016), and recent policy initiatives such as the transition to Sustainability and Transformation Partnerships and the evolution of Integrated Care Systems have led to another wave, with 191 Clinical Commissioning Groups (CCGs) bodies that plan and fund health services for their local areas - merging to become 135 by April 2020 (NHS Digital, 2020).

In terms of recent impetus for mergers, some academics have postulated the emergence of 'metagovernance', whereby Arm's Length Bodies (organisations such as NHS England and NHS Improvement that deliver public services but are not part of government ministries), influence the occurrence of mergers even when they are not aligned with the legal basis of competition under which the NHS currently operates (Hammond et al., 2019). This assertion is supported by the approval of a merger of two NHS hospitals in 2017 by the Competition and Markets Authority, despite them identifying that it would negatively affect competition. The authors conclude that it was given approval because it was coherent with NHS England's policy of integration and collaboration (Hammond et al., 2019). Other literature echoes this, describing a belief by some that NHS Arm's Length Bodies exerted influence to override initial local concerns about a CCG merger (Calovski et al., 2020).

There is some evidence to suggest that mergers can be beneficial if done correctly. Merger can be effective if there is a clear strategic rationale, it is internally generated and there is thorough, early preparation (KPMG, 2011; Dash et al., 2012). Specific benefits include savings of up to $20 \%$ of procurement costs through economies of scale (Dalton, 2014) and additional savings due to rationalisation of services on fewer sites (Dash et al., 2012). Other benefits include the potential for mergers to "buy leaders of challenged providers a little more time... or make it slightly easier to present reconfiguration to the public" (Collins, 2015).

Yet, most evidence suggests that hospital mergers often fail to deliver benefits. Several American studies concluded that mergers do not reduce costs (Dranove and Lindrooth, 2003; Town et al., 2006; Vogt et al., 2006; Harrison, 2011) and a study of NHS hospital mergers concluded that they do not improve key performance indicators such as productivity, care quality and waiting times, with the sole 
improvement in activity and staffing, although this did not lead to improved financial performance (Gaynor et al., 2012). Other work concluded that they damaged clinical services by distracting attention and delaying the development of new services (Fulop et al., 2002). The King's Fund's sobering assessment of NHS mergers found that they are rarely justified, lack evidence, and are unlikely to represent prudent use of public money (Collins, 2015).

This paper evaluates the merger and post-merger integration of two university hospital trusts in the English NHS, Oxford Radcliffe Hospitals (ORH) and the Nuffield Orthopaedic Centre (NOC), which combined to create Oxford University Hospitals (OUH) on $1^{\text {st }}$ November 2011. The evaluation was informed by the two key aims of the merger, which were articulated in the business case developed beforehand (ORH, 2011):

1. To deliver improvements in the quality of clinical care for patients and greater opportunities for staff, by improving care pathways and strengthening the links between academic research, teaching and training and clinical service delivery, in the following areas:
a) Patient outcomes and experience
b) Patient safety
c) Staff development
d) Education and research
e) Specialist services
f) Innovation across the acute and community interface

2. To strengthen long-term financial stability and improve organisational performance, which will enhance the new organisation's ability to obtain Foundation Trust status in line with government requirements.

The evaluation contributes an in-depth case study and practical lessons to the existing literature. Specifically, we contribute an 'instrumental case study' (Stake, 1995) which illuminates three broader phenomena that are currently under-researched.

First, this was a merger of two university teaching hospitals with deep academic links. University hospitals are those with commitment to teaching and research in partnership with a University and can be of any size (University Hospital Association, 2021). This characteristic merits consideration because in addition to integrating clinical services, mergers of university hospitals have to integrate research and education while maintaining the organisational independence of the hospital and university (Pellegrini, 2001; Ovseiko et al., 2015). Organisational culture is a particularly salient factor in merger and post-merger integration in academic medical settings. There are myriad different conceptions of culture, including culture as a compass which provides direction; culture as a glue which binds staff to a common approach; and culture as an exchange-regulator which modulates employee's interactions with each other to reduce friction (Alvesson, 1993). More simply, it can be described as 'the shared ways of thinking, feeling, and behaving in healthcare organisations' (Mannion and Davies, 2018). However, despite evidence describing the association between culture and performance in NHS hospitals (Mannion et al., 2005), its importance is often overlooked (Mallon, 2003; Thier et al., 2014). Therefore, we pay attention to this. 
Second, this was a merger between a generalist trust providing a wide range of clinical services, including some specialised services, and a trust which provided purely specialised services. This is uncommon among acute trust mergers; of the twenty NHS trust mergers between 2010 and 2015 only one other involved a generalist acute trust merging with a specialist trust (Collins, 2015). An in-depth case study of this type of merger is therefore novel.

Finally, the merger is unusual due to the size of the organisations involved. ORH was approximately eight times the size of the NOC in financial terms, and we explore this further. Previous research has concluded that mergers in which the size of the parties significantly differed were no more successful than those in which they were similar (Gaynor et al., 2012), and that while smaller hospitals are under threat internationally, their merger or closure is unlikely to solve problems of costs, care quality, and staffing (Vaughan and Edwards, 2020). However, despite this work we are not aware of case studies which examine this type of merger in depth. 


\section{Methods}

\section{Study setting}

OUH and its legacy hospital trusts ORH and NOC, the old trusts which were dissolved when OUH was formed, are near one another in Oxford, a city in South-East England. Oxford's population of 155,000 is young and diverse, with the fastest turnover in England (Oxford City Council, 2018). The NOC was a small, single-hospital NHS trust specialising in musculoskeletal medicine $(O R H, 2011)$. The ORH was a large, multi-hospital NHS trust providing a wide range of general and specialist services on three sites: the John Radcliffe and Churchill Hospitals in Oxford, and the Horton Hospital in Banbury, Oxfordshire. Before the merger in 2011, the annual turnover of the ORH (£636 million) was approximately eight times that of the NOC ( $f 79$ million). They provided largely non-competing clinical services and formally collaborated in the provision of a small number of complementary services $(\mathrm{ORH}, 2011)$ and in research and education through partnership with Oxford University (Ovseiko et al., 2014b).

\section{Study design}

The study utilized a case study approach. Crowe et al describe a case study as a 'research approach that is used to generate an in-depth, multi-faceted understanding of a complex issue in its real-life context' (Crowe et al., 2011). Case studies have been used extensively to explore 'how, what and why' questions relating to the implementation of new policies or changes to services (Crowe et al., 2011). Therefore, within our case study we aim to explore how the proposed changes were implemented; what the impact of these changes was; and why they resulted in success or failure. In doing so, we focus on practical lessons and insights which may be useful to other healthcare organisations considering merger. Whilst theoretical knowledge is often privileged over practical knowledge, there are strong arguments for the value of practical, context-dependent contributions (Flyvbjerg, 2006).

This study received approval from the University of Oxford's Social Sciences and Humanities Interdivisional Research Ethics Committee (SSD/CUREC1A/13-144). It was designed as a mixed-methods single case evaluation to produce more in-depth and rigorous knowledge. The mixed-methods design combines the strengths and counters the limitations of quantitative and qualitative methods (Moffatt et al., 2006). Qualitative data were collected via one-to-one semi-structured interviews and focus groups and analysed thematically. One-to-one semi-structured interviews are a well-established approach in healthcare research (Grbich, 1999), and were chosen due to their ability to gain a deeper understanding of individuals' experiences and perceptions (McGrath et al., 2019), and the potential they offer to elucidate social phenomena (Silverman, 2010). Practically, one-to-one interviews also offered the interviewee privacy to speak freely. Focus groups were used to gain a broader spectrum of perspectives efficiently, and to use group dynamics to gain a collective view of the research question (Gill et al., 2008). Quantitative data were collected from public documents relating to the merger and were triangulated with the qualitative data. An overview of the study design can be seen in Figure 1 .

\section{Please insert "Figure 1. Study Design" here}

\section{Value co-creation}

To maximise the value of research both in terms of the lessons for policy and practice, as well as for the merged organizations being examined, we used the concept of value co-creation adapted from 
business and management studies (Ramaswamy and Ozcan, 2014; Greenhalgh et al., 2017) but also widely used in healthcare evaluation (Brewster et al., 2015). Namely, we formed a Study Steering Group composed of six executive directors from both legacy trusts and a management consultant who worked on the merger. The Study Steering Group agreed on the goals of the study, guided data collection, oversaw the research, and ensured its validity and relevance to practice.

\section{Sample and data collection}

We collected three types of data for this study. Firstly, qualitative data were collected through 19 individual semi-structured interviews and three focus groups with 17 participants between August 2013 and April 2014 (see Table 1). Starting data collection two and a half years after the merger allowed time for the short-to-medium term impacts to occur, but participants still remembered important details. To design a protocol for interviews and focus groups we reviewed relevant literature and took advice from the Study Steering Group, who assessed it for face validity and relevance to practice.

Participant selection for the individual interviews was via stratified purposive sampling with guidance from the Study Steering Group. Most participants were employees of one of the legacy hospitals prior to merger (either $\mathrm{ORH}$ or the NOC), although the sample also included a patient representative, a management consultant who worked on the merger, and a manager from the Strategic Health Authority (the regional body managing NHS trusts). Most participants continued to be employees of the newly merged organisation (OUH), although three moved to new roles outside the organization after the merger. The sample was stratified by job role (administrative, clinical, or academic) and legacy trust employer (ORH or NOC). It also included representation from several directors from the executive (strategic, operational, and management positions) and non-executive (clinical, academic, and commercial representation) boards of both ORH and NOC. All participants were involved in the merger process to a greater or lesser extent and selected based on the different roles played in this process. For example, the executive directors and the management consultant were directly involved in the day-to-day delivery of the merger process, whereas the manager from the Strategic Health Authority was involved from the perspective of regional NHS policy and strategy.

We also used stratified purposive sampling for focus groups. Interviewees worked for services or departments that had changed during the merger, either due to restructuring (e.g., informatics), or because they previously worked across the boundaries of the two legacy trusts (e.g., spinal surgery; paediatric orthopaedics). The majority were employed by the NOC prior to the merger, and by OUH following the merger. Focus group participants were generally in more junior management positions or purely clinical roles. They were stratified into two groups: clinical and corporate, with separate focus groups for each.

\section{Please insert "Table 1. Study Participants" here}

Interviews and focus groups were conducted on condition of anonymity and only the participants and researchers were present. The one-to-one interviews were conducted in summer and autumn 2013, by two of the authors. The majority took place on site at $\mathrm{OUH}$, but a small number were via telephone. They ranged from approximately thirty minutes to two hours, with an average length of approximately one hour. The focus groups were held in a meeting room at Oxford University Hospitals NHS Trust. They were conducted by two of the authors and lasted for one hour. To minimise bias during data 
collection, respondents were assured that in the presentation of the results they would not be identified or identifiable. For this reason, we replaced actual job titles with generic terms. For example, in September 2011 there were 17 Executive and Non-Executive Directors at ORH. 'Director' is therefore used as a catch-all term to describe multiple possible individuals, making identification of any individual one of them from their quotes alone unlikely. All interviews were digitally recorded and transcribed using a professional transcription service. Informal field notes were occasionally made during the interviews, to note strong emerging themes or remind the interviewer of additional probing questions.

Secondly, quantitative data were collected from public documents from the trust, including annual accounts, annual reports, and board papers; and from NHS access and waiting times standards. Data were analysed to the end of the 2014/15 financial year, three and a half years after the merger. We judged that data collected after this were less likely to be influenced by the merger and more likely to be influenced by extraneous factors such as national policy and the change in OUH leadership in 2015.

Finally, several publicly available key documents relating to the merger were also examined in this study. These were identified by reviewing the 'publications' section of the Trust website for relevant documents published between 2010/11 and 2014/15, and the websites of external organisations such as the Care Quality Commission and NHS England for other relevant documents such as inspection reports and waiting times data. The bulk of documents were analysed after the interviews had been completed with the aim of contextualizing and triangulating interview findings. Such documents included the Oxford University Hospitals' annual reports in the years following the merger, but also other published material evaluating the merger ${ }^{1}$. Additionally, a handful of key documents were also reviewed prior to the primary qualitative data collection to inform the interview schedule, including those outlining the context of the merger or making a business case for it $^{2}$.

\section{Data analysis}

The interviews were analysed first, followed by the documentary analysis. For qualitative data, transcriptions were analysed and coded by two of the authors independently for emerging themes using the process of constant comparison to synthesise and interpret the findings (Straus and Corbin, 1998). They then met to discuss the emerging themes in the context of relevant literature. Another team member read the transcripts independently and suggested revisions. The qualitative data were analysed in paper form.

Our initial coding scheme for the qualitative analysis was organised by three key pre- and post-merger domains, namely, (i) 'performance indicators' (including 'patient outcomes and experiences', 'patient safety', 'financial performance', and 'organisational performance'), (ii) 'staff development', and (iii) 'service impact' (including 'changes in educational and research activities', 'specialist services', and 'innovation across the acute and community interface'). The final themes were agreed by consensus and triangulated with the quantitative data obtained from public documents, as well as the

\footnotetext{
' For example, Stevens, A. (2012), Integration with NOC: Benefits Realisation, Oxford University Hospitals NHS Trust, Oxford

2 For example, ORH (2011), A business case for the integration of the Nuffield Orthopaedic Centre and the Oxford Radcliffe Hospitals. Oxford: Oxford Radcliffe Hospitals NHS Trust
} 
documentary analysis we conducted. Further, in analysing the qualitative data at this stage, we were sensitised by previous research on organisational culture and collaborative working in this setting (Ovseiko and Buchan, 2012; Ovseiko et al., 2014c; Ovseiko et al., 2015). As such, we aimed to identify the assumptions, ideas, beliefs, values, and norms of behaviour associated with organisational culture (Cameron and Quinn, 2006) and how they influenced merger, post-merger integration and collaborative working. These insights were built into the key characteristics of the final coding scheme and have been described as such in the findings. As a final step in the analysis, the Study Steering Group, some of whom were interview participants, reviewed a draft of the findings and provided feedback, although no significant changes were made based on this.

For quantitative data, financial data were analysed using ratio analysis to produce key metrics such as the current ratio and returns on assets. Ratio analysis is commonly used to assess the financial health of organisations (Bruns, 2004), including by boards and regulators in the English NHS (Audit Commission and HFMA, 2010). For example, return on assets was calculated by dividing net income by the assets held in each year, and the current ratio was calculated by dividing current assets by current liabilities for each year. Clinical data are presented descriptively.

For the documentary analysis, key information, both quantitative and qualitative, relating to the merger context, its aims and outcomes was extracted from documents collected from the individual Trust websites and external organizations such as the Care Quality Commission and NHS England. For example, in analysing the Trust's annual reports, key financial metrics for each year were extracted and formed the basis of the financial analysis. In reading CQC inspection reports, key outcomes from inspections were extracted and collated. This was dovetailed with the primary data collected as a means of both corroborating, as well as, complementing the analysis.

\section{Reflexivity}

The interviews and focus groups were conducted by two authors. One of these was formerly a NonExecutive Director of the NOC, whereas the other was an academic junior doctor at OUH. There was a professional relationship between these authors and some study participants prior to the study. While being 'insiders' aided the authors' rapport and trust formation with study participants, they were also cautious about bringing their own subjective experiences and biases to the data collection and analysis process. One mechanism through which they attempted to minimize the effects of these biases were through cross-checking data collection methods and emerging themes with the Study Steering Group. The Study Steering Group was also involved with the formulation of research questions to ensure relevance to practice, and with the selection of participants to ensure that they represented a broad range of knowledge and experience relating to the merger and post-merger integration. On reflection, we do not exclude a possibility that the involvement of the Study Steering Group in the research process may have influenced research questions and data collection towards positive views on the merger and post-merger integration.

All the study participants were interviewed post-hoc and had been involved in some capacity in the merger process, which was one of the key sample selection criteria. The study team was aware of the potential biases that might result from this on the evaluation of the merger and its outcomes and attempted to minimize these by conducting focus groups in addition to one-to-one interviews to both verify and triangulate emerging themes. The information gleaned from the documentary analysis also enabled the team to substantiate the findings emerging from the interviews and focus groups. 


\section{Findings}

The NOC and ORH merged on $1^{\text {st }}$ November 2011. Simultaneously, the Joint Working Agreement between OUH and Oxford University came into being. It provided "a platform for the two organisations to revitalise their partnership in support of the delivery of high quality patient care, research and education" (ORH, 2011). Here, we present the outcomes of the merger organised by key themes which emerged from the analysis.

\section{Outcome of the merger and post-merger integration}

\section{Leadership and strategy}

Interviewees acknowledged that the relationship between the NOC and university was already strong before the merger, with one interviewee concluding that "it's arguably been improved by the merger but it certainly hasn't been damaged ..." (Participant 1, NOC academic clinician). Some interviewees felt that the merger was part of an overarching strategic plan to consolidate Oxford's position as a world-leading centre for medical research and education. OUH became the host organisation for the Oxford Academic Health Science Network (AHSN) in 2013 and in contrast with an unsuccessful application before merger, the government designated the partnership led by the OUH in 2013 as an Academic Health Science Centre (AHSC). Yet, before the merger, the OUH legacy trusts in partnership with the university were awarded the NIHR funding for the Biomedical Research Centre and Unit and were designated with other academic and clinical partners from the Thames Valley as a Health Education and Innovation Cluster (Ovseiko et al., 2014c). There is also quantitative evidence of the improvement of OUH's research performance, as in 2016 the number of active research studies at the trust was $222 \%$ higher than in 2008 (OUH, 2016). Overall, education and research at the interface between the OUH legacy trusts and the university had been on an upward trend long before the merger (Ovseiko et al., 2012; Ovseiko et al., 2014a), and this continued afterwards. Professorial positions and educational curricula were not directly influenced by the merger as these were at the discretion of the University (which was not directly involved in the merger) rather than the hospital trusts.

\section{Organizational structure}

In terms of organizational structure, our results indicated that the merger changed the trust's composition of services compared to the $\mathrm{ORH}$ : "When we came together it was about sixty percent [of general services] and specialist services were forty percent, we're probably the other way around now" (Participant 5, OUH Director). The predominant views regarding specialist services were those of relief and cautious optimism. NOC participants were relieved that the merger did not disrupt the provision of specialist musculoskeletal services: "...all kinds of things being mooted, that we would become an ambulatory centre for the John Radcliffe... our specialist work couldn't be afforded and would have to go... so, the patient committee put up a very large fight about this... And under the terms of the merger the specialist work went on... it seemed so much better than what had been mooted before, I think it came as quite a relief when a merger took place rather than a takeover" (Participant 20, patient representative). Interviewees were also optimistic the strengthened relationship with the university would positively influence the growth of specialist services. 
After the merger, OUH was involved in the ongoing development of an award-winning Emergency Multidisciplinary Unit (EMU) in Oxfordshire. However, it opened in 2010, a year before the merger and resulted from collaboration between the ORH, Oxford Health NHS Foundation Trust, Oxfordshire County Council and Oxford University. It is therefore an example of effective service re-design without merger.

3. Frontline employees

A key indicator for evaluating the effects of the merger on frontline workers was staff development, for which the qualitative data showed a mixed picture. One interviewee felt that opportunities for former NOC staff had improved due to being part of a larger team with greater collective experience: "[NOC staff are] now part of a much wider finance team and have significant opportunities to develop themselves" (Participant 12, former ORH Director). However, another felt that opportunities for former NOC staff were reduced: "It feels much more behind where we were at the NOC in terms of leadership and developing staff... and that feels frustrating, but it also feels exciting, there's opportunity to develop" (Participant 19, former NOC Director). The interviewee cited the NOC's Consultant leadership programme, which was discontinued following the merger. However, other interviewees cited the OUH's nursing academy and nursing band seven development programme as opportunities for enhanced staff development. Quantitative data from the NHS Staff Survey show improving satisfaction before the merger, and continued improvement afterwards with a small decrease in in 2015 (NHS Survey Coordination Centre, 2019).

\section{Operations}

One operational measure against which the merger was evaluated was patient outcomes and experience. We used standardised data from government NHS access and waiting time standards until 2014/15 and Care Quality Commission (CQC) inspection results as a proxy for patient outcomes and experience. Examining access and waiting time standards, compared to ORH's performance prior to merger (2010/11) OUH's performance against the majority of these improved in the year of the merger (2011/12) but declined following this (Figure 2). For example, the Accident \& Emergency (A\&E) four-hour standard (proportion of patients whose emergency care is completed within four hours) performance improved in 2011/12 before declining below the pre-merger level. However, performance against the 62 day wait from screening to cancer treatment target declined in the year of the merger (2011/12) but then increased significantly to delivering it for $96 \%$ of patients in $2012 / 13$.

\section{Please insert “Figure 2. ORH/OUH clinical standards performance, 2010/11-2014/15" here}

The NOC outperformed the ORH on every clinical standard for which we have pre-merger data. It is therefore possible that the OUH's increased performance in the year of the merger was partly due to the addition of the high-performing NOC. Additionally, during this time performance generally declined across the NHS (King's Fund, 2016). Therefore, the later decline in OUH's performance may not be entirely related to internal organisational factors, or the merger. OUH's Care Quality Commission (CQC) inspection results from May 2014 were 'good' overall (CQC, 2014). However, within this the John Radcliffe Hospital was rated as 'requires improvement' (CQC, 2014). The results for the Churchill and Horton General hospitals improved markedly compared to the previous inspection, suggesting that clinical care improved following the merger, although it is impossible to determine 
whether the merger influenced this. Turning to the NOC, in its 2014 inspection ${ }^{3}$, it was rated as 'good' in every area. In the NOC's CQC reports before the merger (NOC, 2010), it was 'fair' in quality of services and 'good' in financial management in 2007/2008, improving to 'good' in both domains in $2008 / 2009$. The NOC was therefore on a trajectory of high performance prior to the merger, which it maintained afterwards. Several changes to patient pathways were aims of the merger (ORH, 2011). These included unifying the NOC and ORH's separate spinal services and providing all paediatric services within the same division, both of which were achieved (Stevens, 2012; OUH, 2018).

A second operational measure against which the merger was evaluated was patient safety. There was a $50 \%$ decline in C.difficile infections following the merger, and the number of MRSA infections remained very low. Between September 2013 and March 2015 there were seven "never events" at OUH. In response to this, an external review (Toft, 2015) concluded that five of the incidents were not genuine "never events" and that "no evidence has been found to suggest that a patient safety problem exists within the Trust..." However, the review did conclude that there was scope for improvement. Furthermore, OUH received a rating of 'requires improvement' in the John Radcliffe's 'safety' domain of its 2014 CQC inspection, suggesting that the Trust had not achieved its aspirations for patient safety by the time of the inspection.

Finally, the OUH made significant progress on electronic prescribing, patient records and admission and discharge information, culminating in the award of 'Digital Hospital of the Year' 2015 (OUH, 2015) and ranking $22^{\text {nd }}$ of 239 organisations in NHS England's 2016 Digital Maturity Index (Illman, 2016). Having met several financial (see below) and clinical goals following the merger (Monitor, 2015), the OUH was awarded Foundation Trust status in 2015.

\section{Financial performance}

Financial performance following the merger was impressive. OUH was in cumulative surplus from 2013/14 and paid back its historic debt, partly reflected by a falling debt ratio (Figure 3 ). The trust's current assets more-than doubled between 2010/11 and 2014/15, whilst current liabilities only increased by approximately $40 \%$, leading to a significant improvement in the trust's current ratio (Figure 3). OUH also used its assets to generate income more effectively, showing a steady increase in its Return on Assets between 2011/12 and 2014/15 (Figure 3), indicating that it achieved the business case aim of enhancing estate utilisation within 1-3 years of the merger. Additionally, in line with the business case identifying savings of $£ 1.6-1.9$ million through reductions in corporate expenditure, at least $£ 1.7$ million was saved (Stevens, 2012). The financial performance of the merged trust was particularly impressive in the context of the wider NHS, as in 2015-16 NHS providers recorded a cumulative deficit of $£ 2.45$ billion, three times higher than the previous year (Dunn et al., 2016). In part, the impressive performance was due to adding the NOC's equity position to the balance sheet (Stevens, 2012), as the NOC had produced surpluses in the years before the merger (NOC, 2011). However, OUH's financial performance continued to improve until 2015, indicating that this was not the sole reason for improved performance.

\footnotetext{
3 Individual hospital sites within larger organizations continue to be inspected individually by the Care Quality Commission after mergers
} 


\section{Please insert "Figure 3. ORH/OUH Financial Ratios, 2010/11-2014/15" here \\ Discussion}

The contingencies that can make mergers a success: Lessons learned

We now reflect on key lessons learned from our case study that other healthcare organisations as well as regulators considering the risks of merger might find useful. Several of these are echoed elsewhere. For example, the 'Making mergers work' report (NHS Improvement, 2016), also concludes that realism, culture change and building momentum are important factors in maximising success. KPMG's analysis of twenty nine mergers supports our findings relating to communication, clinical engagement and culture change being key (KPMG, 2011). Similarly, McKinsey \& Company conclude that it is important to effectively communicate a persuasive clinical case for the merger (Dash et al., 2012). Others find that effective clinical engagement includes more than just clinicians in the board room and must involve those on the front line (Corrigan et al., 2012). Finally, NHS Improvement urges organisations not to underestimate the length of time it can take to achieve improvements (NHS Improvement, 2016).

\section{Develop a strong clinical rationale}

Interviewees strongly believed that mergers should be undertaken to improve patient care and clinical services, not for solely financial or organisational reasons. Having a strong clinical rationale helped engage clinicians and alleviate patients' fears, which in this case were particularly related to a concern that the specialist orthopaedic work at the NOC might cease following the merger. Support from and engagement of staff and patients in the merger process is crucial, and so the importance of a strong clinical rationale should not be underestimated.

\section{Communicate the change strategy widely and early}

To secure the support of staff, communication regarding the merger should start early, be wide across the merging organisations and give staff as much information as possible. Our analysis also indicates that to give mergers the best chance of success, it is best to make changes early. One way of doing this is to create virtual clinical governance and management structures reflecting those which are planned for the new organisation prior to the merger taking place. In our case study this was achieved when the NOC restructured its management team before the merger to mirror that of a clinical division of the $\mathrm{ORH}$, which it would become following the merger. This gave the NOC time to 'bed in' the new management arrangements, resulting in a smoother transition once the merger took place.

3. Increase engagement and collaboration at all levels

In addition to communicating with staff, it is important to engage them in shaping the merger process. As staff develop a sense of ownership of the process, they are likely to improve it by contributing their time and expertise. We found that it is particularly important to identify and persuade key clinicians of the rationale for the merger, because they were seen as opinion leaders who are influential in persuading other members of staff of the benefits of the process.

This case also demonstrates that there should also be collaborative management and clinical leadership across organisational and professional boundaries. Voluntary mergers are only possible if 
managers in both organisations have strong relationships with each other and are trusted by clinicians. The merger between the NOC and ORH was catalysed by a new ORH leadership team who shared the vision of the NOC executive team, and who listened to and acted on clinicians' concerns when required.

\section{Be transparent and realistic about the costs and benefits}

It is important to be transparent and realistic about the anticipated costs and benefits of merger and post-merger integration, or risk staff disillusionment if promises cannot be kept. Organisational changes may be haphazard, and a certain degree of organisational disruption is inevitable. We found a belief that the strategic benefits of mergers take time to develop, and that it's important to be patient when judging the outcome. Specifically, individuals should try to moderate expectations of significant benefits being delivered in the short term and instead focus on the possible long-term strategic gains accrued to the organization due to the merger.

\section{Be sensitive to the feelings of the other organisation}

To form a strong working relationship based on trust, mutual respect and collaboration between individuals from each organisation, individuals in one organisation should be sensitive to the feelings of staff in the other. Our study shows that the discourse of merger should not portray one organisation in a positive light at the expense of the other. The language used to describe the merger is also important for ensuring that all merging organisations engage with the process. For example, the terms "merger", "reconfiguration", "coming together" and "integration" should be preferred to "takeover".

\section{Integrate different organizational cultures effectively}

While merger risks losing some desirable culture traits, it also provides an opportunity to enhance the culture of the merged organisation by drawing on best practice from legacy organisations. In doing so, it is important to understand differences in pre-merger organisational cultures to preserve desirable cultural traits and identify ways of enhancing culture in the merged organisation (Ovseiko et al., 2015). Attempts were made to preserve a "familial" culture at the NOC and to enhance the culture of the merged organisation through a new devolved organisational structure based on clinical divisions and alignment with the university. Organisational development work at ORH also helped to articulate a shared vision and values for the merged organisation and to reflect them in recruitment processes through values-based interviewing $(\mathrm{OUH}, 2011)$.

There should also be a strategic investment in post-merger integration to realise the long-term benefits of the merger. This should be reflected in the strategic plans, organisational development activities, operational priorities, and capital investments of the merged organisation. In that context, merging entities might consider changing the name of the merged organization. A new name helps to establish all parties as meaningful partners in the new organisation. Otherwise, the organisation whose name remains is likely to be perceived as dominant, risking loss of engagement of staff from the other. Additionally, a new name provides a symbolic opportunity to articulate a new vision and to consign stereotypes of the previous organisations to the past. The legacy trusts in this case were perceived rather antagonistically, i.e. a "small and beautiful" yet "not a team player" NOC versus a "big beast on the hill" ORH (Ovseiko et al., 2015). The new name of the merged trust also reflects a shared history and vision of successful academic-clinical collaboration with Oxford University. 


\section{Impact of organizational characteristics on the merger}

Beyond evaluating clinical, financial, and organisational performance, our case study presents a story of a merger with three distinct characteristics based on which we draw several important insights. First, for the merger of two university teaching hospitals, we found a perception that the merger had little short-term impact on academic activity, but that the rationale for the merger was instead focussed on the long-term strategic gains of developing a critical mass of academic expertise in a single location. Nevertheless, we also found that for university teaching hospitals considering merger, it is still important to communicate the clinical rationale for the reorganisation to engage both academic and non-academic clinical staff.

Second, regarding the merger of a generalist trust and a highly specialist trust, the specialist trust had a strong sense of pride and identity, and therefore both the practical and emotional dynamics of the merger needed to be managed sensitively to avoid alienating staff. This was reflected in the expression of "relief" by study participants that the NOC was able to continue as a specialist centre within the new organisation following the merger. We also found that honesty about future organisational changes was felt to be important. Therefore, where a specialist trust is involved in a merger, managers should not make unrealistic promises about its future. Likewise, clinical leadership is important in mergers involving generalist and specialist trusts, particularly in the specialist trust, so that clinical leaders can communicate the rationale for reorganisation.

Third, in terms of the merger of organisations of radically different sizes, leadership in the new organisation should be vigilant about elements of good practice and positive culture within the smaller organisation. In line with previous quantitative research showing differences in organisational culture between the two organisations (Ovseiko et al., 2015), we found challenges for preserving a more positive culture in the smaller organisation as well as opportunities for enhancing the culture of the merged organisation. Specifically, the new organisational structure presented challenges for maintaining a more team-oriented and entrepreneurial culture in the smaller organisation. Likewise, there were adverse effects on informal contacts among staff and accessibility of executives. Yet, the merger provided opportunities to create a more devolved organisational structure across the merged organisation and to catalyse organisational development work. As described above, we found that renaming the new organisation could be an effective technique to avoid staff in the smaller organisation feeling marginalised within the new, larger, organisation. This case study demonstrates the importance of leadership attention being placed on the culture and subcultures of organisations when planning a merger. Furthermore, a deep understanding of the history of the organisations provides critical data and insights as leaders plan a merger.

\section{Limitations}

The study has several limitations. The first limitation is in using national access and waiting time standards as a proxy measure for patient outcomes. Second, due to multiple confounding factors, the limited number of data points in the study period, and a lack of access to raw data we were unable to evaluate the effect of the merger statistically. A third limitation is the relatively small sample size for individual interviews $(n=19)$ and focus groups $(n=17)$, due to resource constraints. Fourth, there is also the possibility of selection bias, as those who agreed to take part may have felt more strongly about the merger than most staff. Fifth, there is also the risk of recollection bias, considering the interviews were conducted almost two years after the merger. This was mitigated by triangulating the one-to- 
one interview data with data from both the focus groups and from documents from the time of the merger. It also needs to be acknowledged that the involvement of the Study Steering Group in the research process may have influenced research questions and data collection towards positive views on the merger and post-merger integration. Finally, because of the single case study design and relatively small sample size, many of our findings are context specific. Therefore, it is imperative to consider the organisational and national context when transferring our findings to other settings. 


\section{Conclusion}

This case study evaluated the outcome of merger and post-merger integration at Oxford University Hospitals and draws lessons for other healthcare organisations and regulators assessing the risks involved when contemplating mergers (de Kam, van Bochove and Bal, 2020). Considering that mergers often fail, this merger was a relative success in terms of organisational, clinical, and financial performance. Overall, there was no marked deterioration but rather mixed improvement in clinical performance as well as strong improvement in financial and organisational performance during the study period. This was delivered through both efficiency gains, by spreading fixed costs over a greater number of patients, and increased diversification through providing a greater range of services, some of which are likely to be more profitable and which also allow the organisation to hedge against the risk of declining revenues in key clinical services.

The results of this study are consistent with an earlier study of the merger process and organisational culture which concluded that the merger was "a necessity, but also one with some promise" (Ovseiko et al., 2015). They reflect the view of competition regulators prior to the merger, who allowed the merger to proceed on the basis of possible clinical and financial benefits, despite an acknowledged adverse impact on choice and competition in elective orthopaedics for which no remedy was made (Sanderson et al., 2017). Indeed, study findings relating to the mixed success of mergers resonate with previous work, which finds that although mergers can be disruptive to everyday hospital operations, they offer practitioners and organisations the opportunity to reflect and learn from each other (de Kam, van Bochove and Bal, 2020).

Yet, we did not find strong evidence to suggest that the benefits of the merger could not have been achieved through other mechanisms, such as collaboration between the two trusts. There have been prominent successes of collaborative networks in the NHS which have used quality improvement methods to improve patient outcomes without a merger (Morris et al., 2014; NHS England, 2015). Moreover, the $\mathrm{ORH}$ may have been capable of addressing its financial and organisational challenges through improved financial planning and allocation by local commissioners. In turn, the NOC was in good financial health at the time of the merger and the perceived challenges to its financial sustainability were hypothetical and predicated on possible future changes to NHS payment systems and judgements by local commissioners. Indeed, at the time of writing there are still three specialist NHS orthopaedic trusts, which are collaborating as part of the National Orthopaedic Alliance (NOA, 2021) through the sharing of best practice and using a shared voice to influence policy issues at national level. Therefore, we urge caution in using mergers to improve clinical, financial, and organisational performance, whilst also suggesting key lessons to maximise the value of a merger should organisations decide that it is the right approach. 


\section{References}

Agrawal, A. and Jaffe, J. F. (2001), "The post-merger performance puzzle", Advances in Mergers \& Acquisitions, Vol. 1 No., pp. 7-41.

Alvesson, M. (1993), Cultural perspectives on organizations, Cambridge University Press.

Audit Commission and HFMA (2010), NHS foundation trust accounts: A guide for non-executives. Revised 2010 edition. London: Audit Commission.

Brewster, L., Aveling, E.L., Martin, G., Tarrant, C., Dixon-Woods, M., Safer Clinical Systems Phase 2 Core Group Collaboration and Writing Committee. (2015), "What to expect when you're evaluating healthcare improvement: a concordat approach to managing collaboration and uncomfortable realities". BMJ Quality \& Safety, 24(5), pp.318-324.

Bruns, W. J. (2004), Introduction to Financial Ratios and Financial Statement Analysis. (Revised September 2004). Harvard Business School Background Note 193-029.

Calovski, V., Taylor, F., O'Shea, A., Brearley, S. and Chambers, M. (2020), Scoping study of the changing landscape of opportunities for patient and public involvement in NHS healthcare commissioning decisionmaking. London: Joint Faculty of Kingston University and St George's University of London.

Cameron, K. S. and Quinn, R. E. (2006), Diagnosing and changing organizational culture: Based on the competing values framework, Jossey-Bass, San Francisco.

Cartwright, S. and Schoenberg, R. (2006), "Thirty years of mergers and acquisitions research: Recent advances and future opportunities", British journal of management, Vol. 17 No. S1, pp. S1-S5.

Collins, B. (2015), Foundation trust and NHS trust mergers. London: The King's Fund.

Corrigan, P., Higton, J. and Morioka, S. (2012), Takeover: Tackling failing NHS hospitals, Reform, London.

CQC (2014), Oxford University Hospitals NHS Trust Quality Report. London: Care Quality Commission.

Crowe, S., Cresswell, K., Robertson, A., Huby, G., Avery, A. and Sheikh, A. (2011), "The case study approach", BMC Medical Research Methodology, Vol. 11 No. 1, pp. 100.

Dalton, D. (2014), Examining new options and opportunities for providers of NHS care: the Dalton review. London: Department of Health.

Dash, P., Meredith, D. and White, P. (2012), Marry in haste, repent at leisure: when do hospital mergers make strategic sense?, McKinsey \& Company, London.

de Kam, D., van Bochove, M. and Bal, R., (2020), "Disruptive life event or reflexive instrument? On the regulation of hospital mergers from a 
quality of care perspective". Journal of health organization and management, Vol, 34 No. 4, pp. 489-503.

Department of Health (2002), A Guide to NHS Foundation Trusts. London: Departmet of Health.

Dranove, D. and Lindrooth, R. (2003), "Hospital consolidation and costs: another look at the evidence", Journal of health economics, Vol. 22 No. 6, pp. 983-997.

Dunn, P., McKenna, H. and Murray, R. (2016), Deficits in the NHS 2016. The King's Fund.

Eisenhardt, K. M. (1989), "Building Theories from Case Study Research", Academy of Management Review, Vol. 14 No. 4, pp. 532-550.

Flyvbjerg, B. (2006), "Five misunderstandings about case-study research", Qualitative Inquiry, Vol. 12 No. 2, pp. 219-245.

Fulop, N., Protopsaltis, G., Hutchings, A., King, A., Allen, P., Normand, C. and Walters, R. (2002), "Process and impact of mergers of NHS trusts: multicentre case study and management cost analysis", BMJ, Vol. 325 No. 7358, pp. 246.

Gaynor, M., Laudicella, M. and Propper, C. (2012), "Can governments do it better? Merger mania and hospital outcomes in the English NHS", Journal of health economics, Vol. 31 No. 3, pp. 528-543.

Giddy, I. (2009), Mergers \& Acquisitons: Definitions and Motivations. New York University.

Gill, P., Stewart, K., Treasure, E. and Chadwick, B. (2008), "Methods of data collection in qualitative research: interviews and focus groups", British Dental Journal, Vol. 204 No. 6, pp. 291-295.

Grbich, C. (1999), Qualitative research in health : an introduction, Sage, London.

Greenhalgh, T., Ovseiko, P. V., Fahy, N., Shaw, S., Kerr, P., Rushforth, A. D., Channon, K. M. and Kiparoglou, V. (2017), "Maximising value from a United Kingdom Biomedical Research Centre: study protocol", Health Research Policy and Systems, Vol. 15 No. 1, pp. 70.

Hammond, J., Speed, E., Allen, P., McDermott, I., Coleman, A. and Checkland, K. (2019), "Autonomy, accountability, and ambiguity in arm's-length meta-governance: the case of NHS England", Public Management Review, Vol. 21 No. 8, pp. 1148-1169.

Harrison, T. D. (2011), "Do mergers really reduce costs? Evidence from hospitals", Economic inquiry, Vol. 49 No. 4, pp. 1054-1069.

Illman, J. (2016), Revealed: First digital maturity index scores in full. Health Service Journal. London.

King's Fund. (2016), "Quarterly Monitoring Report", available at: http://amr.kingsfund.org.uk/2016/18/data (accessed 21 May 2018).

King, D. R., Dalton, D. R., Daily, C. M. and Covin, J. G. (2004), "Meta-analyses of post-acquisition performance: indications of unidentified moderators", Strategic Management Journal, Vol. 25 No. 2, pp. 187-200.

KPMG (1999), Unlocking the shareholder value: the keys to success. Mergers \& acquisitions, a global research report, KPMG, London. 
KPMG (2011), Taking the Pulse: A global study of mergers and acquisitions in Healthcare, KPMG, London.

Mallon, W. T. (2003), "The alchemists: a case study of a failed merger in academic medicine", Academic Medicine, Vol. 78 No. 11, pp. 10901104.

Mannion, R. and Davies, H. (2018), "Understanding organisational culture for healthcare quality improvement", BMJ, Vol. 363 No., pp. k4907.

Mannion, R., Davies, H. T. and Marshall, M. N. (2005), "Cultural characteristics of "high" and "low" performing hospitals", J Health Organ Manag, Vol. 19 No. 6, pp. 431-9.

McGrath, C., Palmgren, P. J. and Liljedahl, M. (2019), "Twelve tips for conducting qualitative research interviews", Medical Teacher, Vol. 41 No. 9, pp. 1002-1006.

Moffatt, S., White, M., Mackintosh, J. and Howel, D. (2006), "Using quantitative and qualitative data in health services research - what happens when mixed method findings conflict? [ISRCTN61522618]", BMC Health Serv Res, Vol. 6 No. 28, pp. 1-10.

Monitor. (2015), "Oxford University Hospitals awarded foundation trust status by regulator", available at: https://www.gov.uk/government/news/oxford-university-hospitalsawarded-foundation-trust-status-by-regulator (accessed 21 May 2018).

Morris, S., Hunter, R. M., Ramsay, A. I. G., Boaden, R., McKevitt, C., Perry, C., Pursani, N., Rudd, A. G., Schwamm, L. H., Turner, S. J., Tyrrell, P. J., Wolfe, C. D. A. and Fulop, N. J. (2014), "Impact of centralising acute stroke services in English metropolitan areas on mortality and length of hospital stay: difference-in-differences analysis", BMJ, Vol. 349 No. g4757 pp. 1-10.

NOA (2021), "National Orthopaedic Alliance", available at: https://nationalorthopaedicalliance.co.uk/ (accessed 18 Oxtober 2021).

NHS Digital. (2020), "Change summary - 2020 STP, CCG and Commissioning Hub reconfiguration", available at:

https://digital.nhs.uk/services/organisation-data-service/changesummary---stp-reconfiguration (accessed 7 November 2020).

NHS England (2015), National Peer Review Report: Major Trauma 2015. London: NHS England.

NHS Improvement (2016), Making mergers work. London: NHS Improvement. NOC (2010), Annual Report 2009-2010. Oxford: Nuffield Orthopaedic Centre NHS Trust.

NOC. (2011), "Annual Reports Archive: Nuffield Orthopaedic Centre NHS Trust", available at: http://www.ouh.nhs.uk/about/publications/reportarchive.aspx\#noc (accessed 21 May 2018).

$\mathrm{ORH}$ (2011), A business case for the integration of the Nuffield Orthopaedic Centre and the Oxford Radcliffe Hospitals. Oxford: Oxford Radcliffe Hospitals NHS Trust. 
OUH. (2011), "Vision and values", available at:

https://www.ouh.nhs.uk/about/vision-and-values/default.aspx

(accessed 20 May 2018).

OUH. (2015), "Trust named Digital Hospital of the Year 2015", available at: http://www.ouh.nhs.uk/news/article.aspx?id=365 (accessed 21 May 2018).

OUH. (2016), "Record high for Oxford University Hospitals research studies", available at: https://www.ouh.nhs.uk/news/article.aspx?id=553 (accessed 21 May 2018).

OUH. (2018), "Welcome to the Oxford Spinal Unit", available at: https://www.ouh.nhs.uk/spinal/ (accessed 21 May 2018).

Ovseiko, P. V. and Buchan, A. M. (2012), "Organizational culture in an academic health center: an exploratory study using a Competing Values Framework", Acad Med, Vol. 87 No. 6, pp. 709-718.

Ovseiko, P. V., Davies, S. M. and Buchan, A. M. (2014a), "AM last page: funding of academic research in clinical medicine in the United Kingdom", Acad Med, Vol. 89 No. 5, pp. 830.

Ovseiko, P. V., Heitmueller, A., Allen, P., Davies, S. M., Wells, G., Ford, G. A., Darzi, A. and Buchan, A. M. (2014b), "Improving accountability through alignment: the role of academic health science centres and networks in England", BMC Health Serv Res, Vol. 14 No. 24, pp. 1-14.

Ovseiko, P. V., Melham, K., Fowler, J. and Buchan, A. M. (2015),

"Organisational culture and post-merger integration in an academic health centre: a mixed-methods study", BMC Health Serv Res, Vol. 15 No. 25, pp. 1-14.

Ovseiko, P. V., O'Sullivan, C., Powell, S. C., Davies, S. M. and Buchan, A. M. (2014c), "Implementation of collaborative governance in cross-sector innovation and education networks: evidence from the National Health Service in England", BMC Health Serv Res, Vol. 14 No. 552, pp. 114.

Ovseiko, P. V., Oancea, A. and Buchan, A. M. (2012), "Assessing research impact in academic clinical medicine: a study using Research Excellence Framework pilot impact indicators", BMC Health Serv Res, Vol. 12 No. 478, pp. 1-23.

Oxford City Council. (2018), "Oxford's Population", available at:

https://www.oxford.gov.uk/info/20131/population/459/oxfords_populat ion (accessed 21 May 2018).

Pellegrini, V. D., Jr. (2001), "Mergers involving academic health centers: a formidable challenge", Clinical Orthopaedics and Related Research, Vol. 391 No. Oct, pp. 288-296.

Petit, B. S. and Ferris, K. R. (2013), Valuation for Mergers and Acquisitions FT Press, London.

Ramaswamy, V. and Ozcan, K. (2014), The co-creation paradigm, Stanford University Press, Stanford.

Sanderson, M., Allen, P. and Osipovic, D. (2017), "The regulation of competition in the National Health Service (NHS): what difference has 
the Health and Social Care Act 2012 made?", Health Economics, Policy and Law, Vol. 12 No. 1, pp. 1-19.

Silverman, D. (2010), Doing qualitative research : a practical handbook, SAGE, London.

Stake, R. E. (1995), The art of case study research : perspectives on practice, Sage, Thousand Oaks, Calif. ; London.

Stevens, A. (2012), Integration with NOC: Benefits Realisation, Oxford University Hospitals NHS Trust, Oxford.

Straus, A. and Corbin, J. (1998), Basics of Qualitative Research: Techniques and Procedures for Developing Grounded Theory, Sage, London.

Thier, S. O., Kelley, W. N., Pardes, H., Knight, A. W. and Wietecha, M. (2014), "Success factors in merging teaching hospitals", Acad Med, Vol. 89 No. 2, pp. 219-23.

Toft, B. (2015), External Review of 'Never Events' that occurred at Oxford University Hospitals NHS Trust during the period 13 September 2013 to 26 March 2015 OUH, Oxford.

Town, R., Wholey, D., Feldman, R. and Burns, L. R. (2006), The welfare consequences of hospital mergers. NBER Working Paper No. 12244. Cambridge, MA: National Bureau of Economic Research.

University Hospital Association (2021), "University Hospital Status", London: Univversity Hospital Association, available at: https://www.universityhospitals.org.uk/wpcontent/uploads/2021/09/University-Hospital-Status-2021.pdf (accessed 18 October 2021).

Vaughan, L. and Edwards, N. (2020), "The problems of smaller, rural and remote hospitals: Separating facts from fiction", Future Healthcare Journal, Vol. 7 No. 1, pp. 38-45.

Vogt, W. B., Town, R. and Williams, C. H. (2006), How has hospital consolidation affected the price and quality of hospital care?, Robert Johnson Wood Foundation, Princeton, NJ. 


\begin{tabular}{|c|c|c|c|c|c|c|}
\hline \multicolumn{7}{|c|}{ Individual Interviews* } \\
\hline Profession & Clinician & Administrator & Academic & $\begin{array}{l}\text { Patient } \\
\text { Representative }\end{array}$ & $\begin{array}{l}\text { Total, including } \\
\text { combined roles }\end{array}$ & $\begin{array}{l}\text { Total individual } \\
\text { interviewees }\end{array}$ \\
\hline 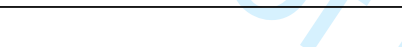 & 9 & 16 & 4 & 1 & 30 & 19 \\
\hline $\begin{array}{l}\text { Pre-Merger } \\
\text { Organisational } \\
\text { Affiliation }\end{array}$ & $\begin{array}{l}\text { Oxford } \\
\text { Radcliffe } \\
\text { Hospitals }\end{array}$ & $\begin{array}{l}\text { Nuffield } \\
\text { Orthopaedic } \\
\text { Centre }\end{array}$ & Other & & & Total \\
\hline & 7 & 10 & 2 & & & 19 \\
\hline \multicolumn{7}{|c|}{ Focus Groups } \\
\hline & Clinician & Administrator & Academic & $\begin{array}{l}\text { Patient } \\
\text { Representative }\end{array}$ & $\begin{array}{l}\text { Total, including } \\
\text { combined roles: }\end{array}$ & $\begin{array}{l}\text { Total individual } \\
\text { interviewees: }\end{array}$ \\
\hline Clinical Focus Group & 8 & 0 & 1 & 0 & 9 & 8 \\
\hline $\begin{array}{l}\text { Corporate Focus } \\
\text { Group }\end{array}$ & 0 & 9 & 0 & 0 & 0 & 9 \\
\hline
\end{tabular}

*NB: Three participants were a clinician, administrator and academic; one participant was both a clinician and an academic; four participants were both clinicians and administrators; and eleven participants had single roles 


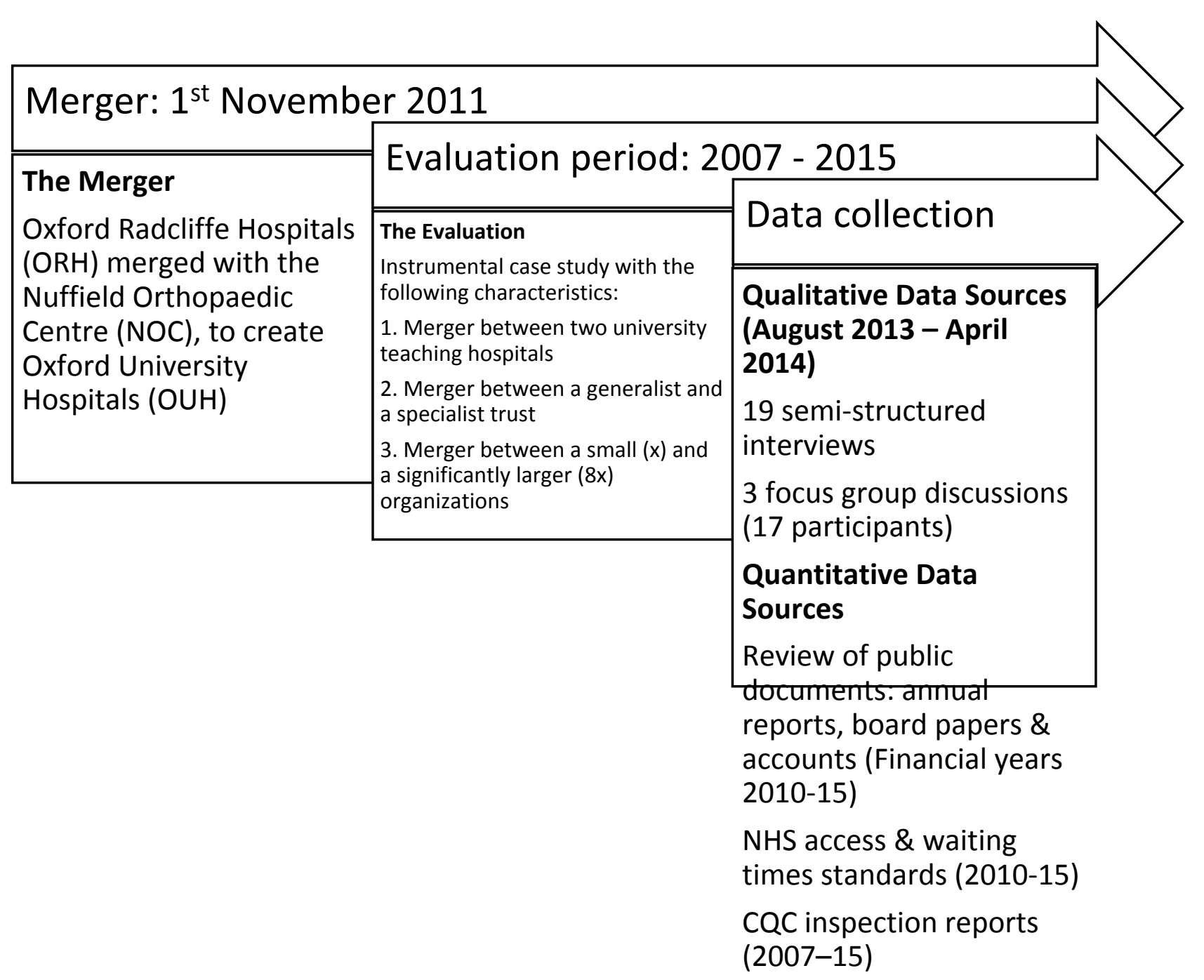


2010/11

2011/12

2012/13

2013/14

2014/15

A\&E 4 Hour Standard Cancer Two Week Wait Standard Admitted 18 Wk Referral to Treatment

95.13\%

$95.60 \%$

$92.90 \%$

$93.23 \%$

$89.80 \%$
$87.40 \%$

$96.68 \%$

$95.43 \%$

$95.62 \%$

$94.90 \%$
$78 \%$

$89.76 \%$

$91.92 \%$

$88.78 \%$

$86.85 \%$

\section{ORH/OUH Clinical Standards Performance}

$100.00 \%$

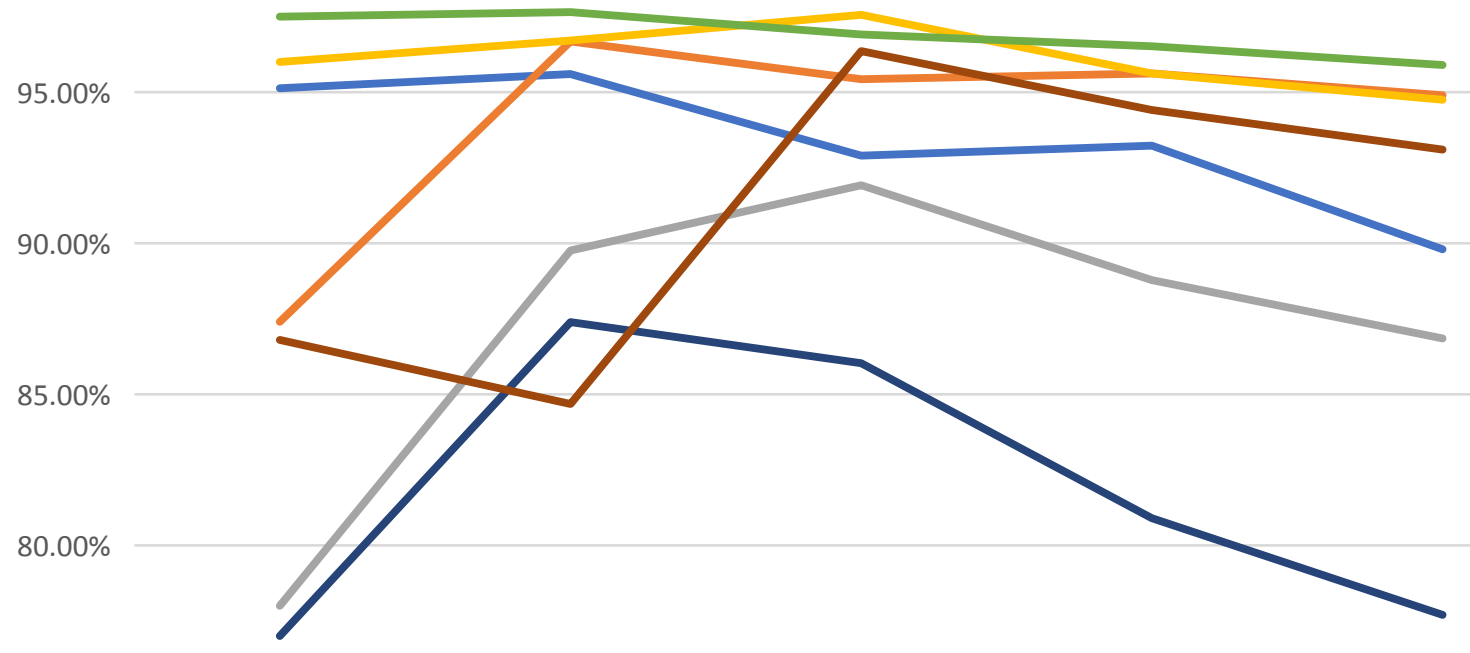

$75.00 \%$

$2011 / 12$

2012/13

$2013 / 14$ 


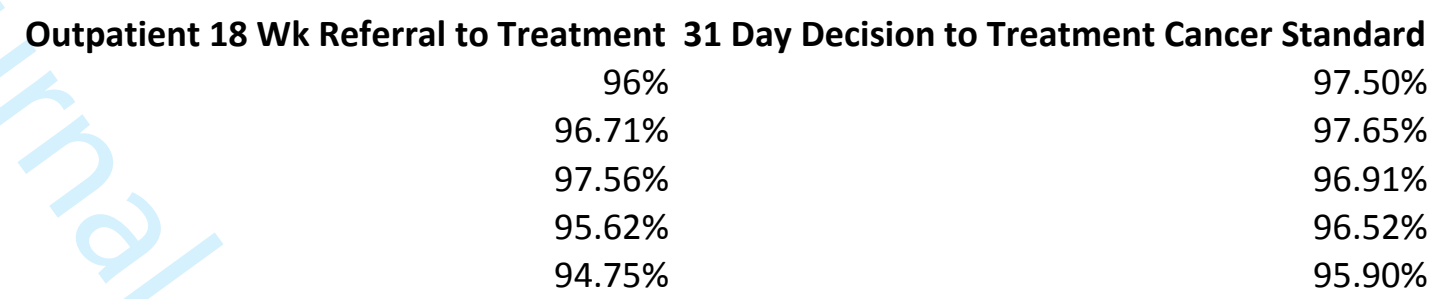

A\&E 4 Hour Standard

Cancer 2 Wk Wait Standard

Admitted 18 Wk Referral to Treatment

Outpatient 18 Wk Referral to Treatment

31 day decision to

treatment cancer standard

62 Day Referral to

Treatment Cancer Standard

62 Day Screening to

Treatment Cancer Standard

15 


$\begin{array}{rr}62 \text { Day Referral to Treatment Cancer Standard } & 62 \text { Day Screening to Treatment Cancer Standard } \\ 77 \% & 86.80 \% \\ 87.39 \% & 84.68 \% \\ 86.03 \% & 96.36 \% \\ 80.90 \% & 94.41 \% \\ 77.70 \% & 93.10 \%\end{array}$




\begin{tabular}{lrrrrr} 
& Quick Ratio & Return on Assets & \multicolumn{2}{c}{ Current Ratio } & \multicolumn{2}{c}{ Debt Ratio } \\
$\mathbf{2 0 1 0 / 1 1}$ & 0.401089452 & 1.120186749 & 0.522063266 & 0.589139569 \\
$\mathbf{2 0 1 1 / 1 2}$ & 0.638081926 & 0.984600549 & 0.739425658 & 0.535368273 \\
$\mathbf{2 0 1 2 / 1 3}$ & 0.750313605 & 1.030572073 & 0.842193861 & 0.534212351 \\
$\mathbf{2 0 1 3 / 1 4}$ & 0.844664487 & 1.042659981 & 0.934665782 & 0.507904488 \\
$\mathbf{2 0 1 4 / 1 5}$ & 0.848942261 & 1.131322323 & 0.951679118 & 0.528036658
\end{tabular}

ORH/OUH Financial Ratios

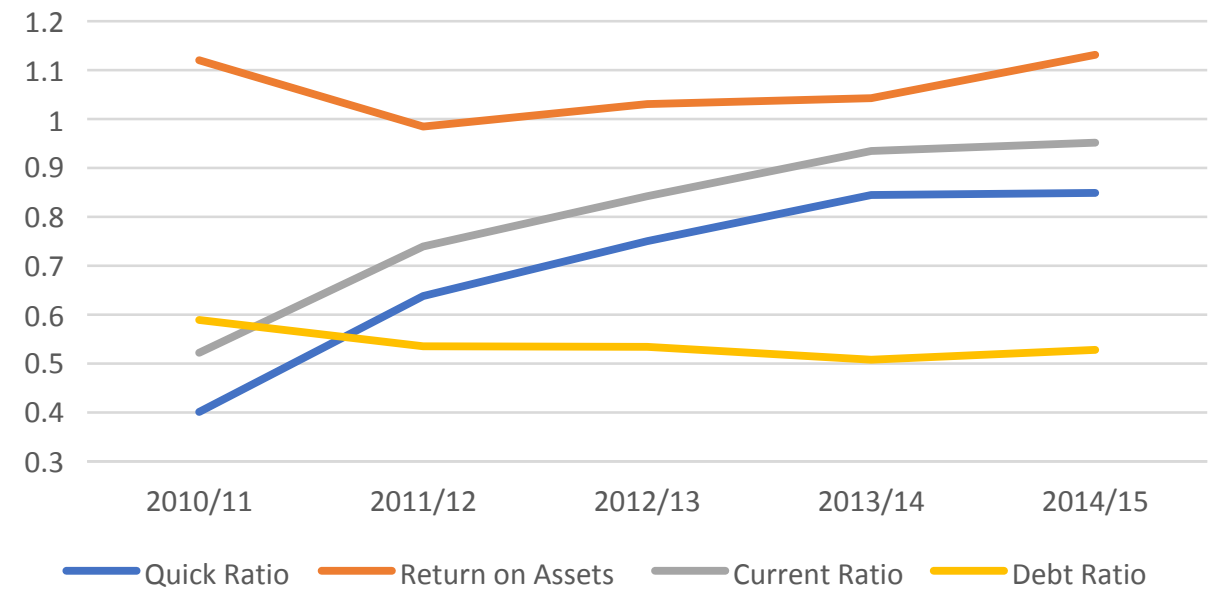

